\title{
Two-Particle Lattice Green Functions on a Two-Dimensional Rectangular Lattice with Next-Nearest Neighbor Hopping
}

\author{
M. Bak
}

Received: 8 August 2011 / Accepted: 18 October 2011 / Published online: 5 November 2011

(C) The Author(s) 2011. This article is published with open access at Springerlink.com

\begin{abstract}
The paper shows a way to find two-particle lattice Green functions (LGF) on any site of a two-dimensional, rectangular lattice with hopping to nearest- and next-nearest neighbors. Exact, analytical formulas using elliptic integrals for the on-site- $G_{0,0}$, nearest neighbor- $G_{0,1}$ and $G_{1,0}$ and next-nearest neighbor- $G_{1,1}$ LGF's are shown. Difference equation for general $G_{m, n}$ is given together with five kind of recurrence relations among LGF's and their derivatives. A way of assembling recurrence relations into closed sets of equations, enabling to find $G_{n, m}$ on any lattice site (requiring the knowledge of five parameters) is described. The differences between one- and two-particle LGF's are shown.
\end{abstract}

Keywords Lattice Green functions · Elliptic integrals

\section{Introduction}

The theory of Green functions has been applied in physics for a long time. It has been used, e.g., in quantum mechanics, calculations of many-body systems, quantum statistical mechanics, condensed matter physics to name a few. In condensed matter field the lattice Green functions (LGF) are more widely applied [1]. They have the general form of Watson integrals [2], which appeared in physics during the research of magnetism in the sixties (particularly Heisenberg model on three dimensional (3d) lattices [3]). Apart from that, LGF's have also found use, e.g., in lattice statistical problems, random walks and percolation theories [4], problems of transport and diffusion in solids [5], theories of impurities in solids [6], lattice dynamics [7], investigations of tight-binding models on the lattice [8], investigations of electron band structures in crystals [9], models of phase transition in particular in $2 \mathrm{~d}$ and in calculations of resistivity and capacitance in infinite networks of resistors or capacitors [10-13].

The latter field is of much interest in connection with high temperature superconductivity (HTSC), which is widely believed to be caused by Bose condensation of tightly-bound

M. Bak (凶)

Dep. of Pysics, A. Mickiewicz University, ul. Umultowska 85, 61-614 Poznan, Poland e-mail:mbak@amu.edu.pl 
electron pairs. Properties of a single pair can be calculated (exactly!) by lattice Green functions [14-18]. Collection of such pairs can be regarded as incoherent, low density limit of tightly-bound electron pairs' condensate; in case of $s$-wave pairing the threshold for pairs' appearance is also a threshold for $s$-wave superconductivity to appear [19]. It is also shown, that phase of uncorrelated pairs do exist in the phase diagram of $t-J$ model [20].

After practical realization of Hubbard model in the optical lattices of cold atoms, comparison of experiment with such exact solutions can help to gain much deeper insight into the tight-binding models' physics than was possible so far.

LGF's concerning Hamiltonians with nearest-neighbor (nn) hopping have been quite well known, though mostly for 3d lattices, since seventies [21, 22]. Yet in view of possible application to investigations of HTSC, where next-nearest hopping (nnn) can be substantial, a need appears to calculate nnn LGF's in $2 d$ rectangular lattice. To be able to apply the results to the calculations of bound (and resonant) pairs we need a two-particle LGF. The current paper presents just that.

The available $2 \mathrm{~d}$ results are scarce: the imaginary part of one-particle LGF's with nnn, i.e., the density of states, is known [7, 23, 24] (yet not sufficient to calculate, e.g., bound states, where also the real part is needed and also outside the band). The full one-particle LGF with nnn hopping was given lately in Ref. [25]. A recursion formula for peculiar LGF on a 2d lattice was given by Morita [26] (but not the LGF itself). Most of the known results concern $n$ hopping and one-particle functions, e.g., Ref. [27]. The present paper can be regarded as an extension of [27] and its reformulation in the language of elliptic integrals (in [27] hypergeometric functions are used).

The plan of work is as follows: in Sect. 2 the basic definitions concerning LGF's for tightbinding Hamiltonians with nn and nnn hopping are presented. Section 3 shows a detailed analytical solution of the most basic LGF- $G_{0,0}$ and the difference between one- and twoparticle Green functions. The formulae for $G_{0,1}, G_{1,0}, G_{1,1}$, needed to find any other LGF, are shown in Sect. 4. Section 5 presents 5 kind of recursion relations, including also relations among LGF's derivatives, and explains a special way in which they have to be assembled to make a closed system of equations. The paper is closed by discussion and conclusions in Sect. 6.

\section{Definitions}

We begin with the general definition of the Green function:

$$
(E-\mathbf{H}(\mathbf{r})) G\left(E ; \mathbf{r}, \mathbf{r}^{\prime}\right)=\delta\left(\mathbf{r}-\mathbf{r}^{\prime}\right),
$$

where $E$ is complex variable and $\mathbf{H}$ is linear, hermitian, differential operator. Thus $G$ can be described as a resolvent:

$$
G=\frac{\mathbf{1}}{E-\mathbf{H}}=\sum_{n} \frac{\left|\phi_{n}\right\rangle\left\langle\phi_{n}\right|}{E-E_{n}},
$$

where $\left|\phi_{n}\right\rangle$ form complete set of orthonormal eigenstates and $E_{n}$ are eigenvalues of operator $\mathbf{H}$. If $\mathbf{H}$ is kinetic part of tight binding Hamiltonians (see, e.g. [1]):

$$
\mathbf{H}=\sum_{\langle i, j\rangle\rangle} \sum_{\sigma} t_{i, j} c_{i, \sigma}^{\dagger} c_{j, \sigma},
$$

where $t_{i, j}$ is hopping integral, $c_{i, \sigma}^{\dagger}$ and $c_{j, \sigma}$ are creation and annihilation operators of electrons with spin $\sigma$ on sites $i$ and $j$ respectively, which are nn or nnn sites (that is meant by 
$\langle\langle\cdot\rangle\rangle)$ of infinite, two-dimensional, rectangular lattice. One-particle eigenstates of Hamiltonian (3) are states with fixed (quasi)momentum $\mathbf{k}$, which belong to the reciprocal space: thus eigenvectors can be denoted as $|\mathbf{k}\rangle$. Their real-space representations are just Bloch waves. Eigenenergy of state $|\mathbf{k}\rangle$ on a two-dimensional rectangular lattice with nnn hopping is given as:

$$
E_{\mathbf{k}}=2 t_{x} \cos \left(k_{x} a_{x}\right)+2 t_{y} \cos \left(k_{y} a_{y}\right)+4 t_{2} \cos \left(k_{x} a_{x}\right) \cos \left(k_{y} a_{y}\right),
$$

where $a_{x}$ and $a_{y}$ are lattice constants in $x$ and $y$ directions respectively, $k_{x}$ and $k_{y}$ are components of vector $\mathbf{k}, t_{x}, t_{y}$ are nn hopping integrals in $x$ and $y$ directions resp., and $t_{2}$ is hopping integral between next-nearest neighbors. The one-particle Green function finally takes the form:

$$
G\left(E ; \mathbf{r}, \mathbf{r}^{\prime}\right)=\frac{1}{N} \sum_{\mathbf{k}} \frac{e^{i \mathbf{k}\left(\mathbf{r}-\mathbf{r}^{\prime}\right)}}{E-E_{\mathbf{k}}} .
$$

Its imaginary part (in the sense of $\lim _{\epsilon \rightarrow 0} E+i \epsilon$ ) yields density of states for a given lattice; $N$ denotes number of lattice sites.

The two-particle Green function operator can be written as:

$$
G(E)=\frac{1}{N} \sum_{\mathbf{k}_{1}} \sum_{\mathbf{k}_{2}} \frac{\left|\mathbf{k}_{1}, \mathbf{k}_{2}\right\rangle\left\langle\mathbf{k}_{1}, \mathbf{k}_{2}\right|}{E-E_{\mathbf{k}_{1}}-E_{\mathbf{k}_{2}}},
$$

where $\left|\mathbf{k}_{1}, \mathbf{k}_{2}\right\rangle$ are the two-particle eigenstates of operator $\mathbf{H}$, (3). It is useful to change the basis to $|\mathbf{K}, \delta \mathbf{k}\rangle$ instead of $\left|\mathbf{k}_{1}, \mathbf{k}_{2}\right\rangle$, where $\mathbf{K}=\mathbf{k}_{1}+\mathbf{k}_{2}$ is total (center-of-mass) momentum and $\delta \mathbf{k}=\left(\mathbf{k}_{1}-\mathbf{k}_{2}\right) / 2$ is relative momentum. As $\mathbf{H}$, (3), conserves the total momentum, we can consider Green functions for each $\mathbf{K}$ separately:

$$
G_{\mathbf{K}}\left(E ; \delta \mathbf{r}, \delta \mathbf{r}^{\prime}\right)=\frac{1}{N} \sum_{\delta \mathbf{k}} \frac{e^{i \delta \mathbf{k}\left(\delta \mathbf{r}-\delta \mathbf{r}^{\prime}\right)}}{E-E_{\mathbf{K} / 2-\delta \mathbf{k}}-E_{\mathbf{K} / 2+\delta \mathbf{k}}},
$$

where $\delta \mathbf{r}=\mathbf{r}_{1}-\mathbf{r}_{2}$ denotes relative position between two particles in a pair. While the numerator of the above two-particle Green function is formally the same as in one-particle case-(5), the denominator got more complicated:

$$
\begin{aligned}
E_{\mathbf{K} / 2-\delta \mathbf{k}}+E_{\mathbf{K} / 2+\delta \mathbf{k}}= & \gamma_{1} \cos \left(\delta k_{x} a_{x}\right)+\gamma_{2} \cos \left(\delta k_{y} a_{y}\right) \\
& +\gamma_{3} \cos \left(\delta k_{x} a_{x}\right) \cos \left(\delta k_{y} a_{y}\right)+\gamma_{4} \sin \left(\delta k_{x} a_{x}\right) \sin \left(\delta k_{y} a_{y}\right),
\end{aligned}
$$

where

$$
\begin{aligned}
& \gamma_{1}=4 t_{x} \cos \left(K_{x} a_{x} / 2\right), \\
& \gamma_{2}=4 t_{y} \cos \left(K_{y} a_{y} / 2\right), \\
& \gamma_{3}=8 t_{2} \cos \left(K_{x} a_{x} / 2\right) \cos \left(K_{y} a_{y} / 2\right), \\
& \gamma_{4}=8 t_{2} \sin \left(K_{x} a_{x} / 2\right) \sin \left(K_{y} a_{y} / 2\right) .
\end{aligned}
$$

After comparing with (4), we can see that apart from constant multipliers an additional term proportional to $\sin \left(k_{x} a_{x}\right) \sin \left(k_{y} a_{y}\right)$ appeared on the r.h.s. of (8). This term is responsible for the qualitative difference between the one- and two-particle LGF's, which become the same (up to a constant) only for $\mathbf{K}=0$. Eventually a Green function for a fixed total momentum ( $\mathbf{K}$ index is omitted henceforth in the notation), for $\delta \mathbf{r}-\delta \mathbf{r}^{\prime}$ being a lattice vector: $\left(m a_{x}, n a_{y}\right)$, after trivial variable change $k_{s} a_{s} \rightarrow k_{s}, s \in\{x, y\}$, can be written down in the infinite lattice limit as an integral: 


$$
\begin{aligned}
& G_{m, n}(E) \\
& \quad=\frac{1}{(2 \pi)^{2}} \int_{-\pi}^{\pi} d k_{x} \int_{-\pi}^{\pi} d k_{y} \frac{e^{i\left(k_{x} m+k_{y} n\right)}}{E-\gamma_{1} \cos k_{x}-\gamma_{2} \cos k_{y}-\gamma_{3} \cos k_{x} \cos k_{y}-\gamma_{4} \sin k_{x} \sin k_{y}} .
\end{aligned}
$$

In fact, numerator can be replaced by $\cos \left(k_{x} m+k_{y} n\right)$, as the part of the integral with the $\sin \left(k_{x} m+k_{y} n\right)$ disappears on rectangular lattice due to symmetry. For the same reasons we can not narrow down the integration to $\int_{0}^{\pi} \int_{0}^{\pi} d k_{x} d k_{y}$.

\section{Analytic Solution for $G_{0,0}$}

We begin with finding the solution of the simplest of Green functions of the type given by (13), namely $G_{0,0}$. After standard $u=\tan \left(k_{x} / 2\right)$ substitution, integral over $k_{x}$ is replaced by integral over quadratic polynomial in $u$ in the denominator. For negative discriminant of that polynomial the integral can be expressed by arctan, while for the positive-by log. The calculation of definite integral and second substitution $v=\tan \left(k_{y} / 2\right)$, leaves us with the integral over the inverse of the square root of the negative of the above-mentioned discriminant. If the (bare) discriminant is negative, then we must still multiply the whole formula under the integral by the sign of the leading term of the polynomial in $u$ (which will now depend on $v$ )-because the arctan depended on that sign:

$$
G_{0,0}(E)=\frac{1}{(\pi)^{2}} \int_{-\infty}^{\infty} \frac{\operatorname{sig}_{Q}[f(v)]}{\sqrt{Q(v)}} d v
$$

where:

$$
\begin{aligned}
& Q(v)=a v^{4}+b v^{2}+c, \\
& a=\left(E+\gamma_{2}\right)^{2}-\left(\gamma_{1}-\gamma_{3}\right)^{2}, \\
& b=2\left(E^{2}-\gamma_{1}^{2}-\gamma_{2}^{2}+\gamma_{3}^{2}-2 \gamma_{4}^{2}\right), \\
& c=\left(E-\gamma_{2}\right)^{2}-\left(\gamma_{1}+\gamma_{3}\right)^{2}, \\
& f(v)=E+\gamma_{1}+\left(\gamma_{3}-\gamma_{2}\right) \frac{1-v^{2}}{1+v^{2}}, \\
& \operatorname{sig}_{Q}[x]= \begin{cases}\operatorname{sign}[x], & \text { for } Q(v) \geq 0 \\
1, & \text { for } Q(v)<0 .\end{cases}
\end{aligned}
$$

Note, that $f(v)$ may become zero and change the sign for $v=v_{E}=\sqrt{v_{E}^{2}}$ if $v_{E}^{2}>0$ :

$$
v_{E}^{2}=\frac{-E-\gamma_{1}+\gamma_{2}-\gamma_{3}}{E+\gamma_{1}+\gamma_{2}-\gamma_{3}}
$$

The sign $[f(v)]$ can be simplified to:

$$
\operatorname{sign}[f(v)]= \begin{cases}\operatorname{sign}\left[\alpha \cdot\left(v^{2}-v_{E}^{2}\right)\right], & v_{E}^{2}>0 \Longleftrightarrow\left|E+\gamma_{1}\right|<\left|\gamma_{3}-\gamma_{2}\right| \\ \operatorname{sign}\left[E+\gamma_{1}\right], & v_{E}^{2}<0 \Longleftrightarrow\left|E+\gamma_{1}\right|>\left|\gamma_{3}-\gamma_{2}\right|,\end{cases}
$$

where $\alpha=\operatorname{sign}\left[\gamma_{2}-\gamma_{3}\right]$. Condition $v_{E}^{2}<0$ means, that $f(v)$ does not change sign for any $v$. To proceed we need the roots of $Q(v)$ : 


$$
v_{1,2}^{2}=\frac{\gamma_{1}^{2}+\gamma_{2}^{2}-\gamma_{3}^{2}+2 \gamma_{4}^{2}-E^{2} \pm 2 \sqrt{\left(\gamma_{1} \gamma_{2}+E \gamma_{3}\right)^{2}+\left(\gamma_{1}^{2}+\gamma_{2}^{2}-\gamma_{3}^{2}-E^{2}\right) \gamma_{4}^{2}+\gamma_{4}^{4}}}{\left(\gamma_{1}+\gamma_{2}-\gamma_{3}+E\right)\left(-\gamma_{1}+\gamma_{2}+\gamma_{3}+E\right)},
$$

where $v_{1}^{2}$ and $v_{2}^{2}$ denote the solutions with the opposite signs at the square root. The Green function now can be written as:

$$
G_{0,0}(E)=\frac{2}{\pi} \int_{0}^{\infty} \frac{\operatorname{sig}_{Q}[f(v)]}{\sqrt{a\left(v^{2}-v_{1}^{2}\right)\left(v^{2}-v_{2}^{2}\right)}} d v
$$

what can be easily cast into the standard form of elliptic integral, with the denominator: $\sqrt{\left(1-v^{2}\right)\left(1-k^{2} v^{2}\right)}$, yet the need to resolve the signum in the numerator makes the form (24) more feasible.

As each positive root $v_{1,2}^{2}$ causes singularity of the integrand of (24) the signs of roots (23) are of basic importance and are used to identify the solutions. Green function for complex roots $v_{1}^{2}=\left(v_{2}^{2}\right)^{*} \equiv v_{c}^{2}$ will be denoted as $G^{C}$; for real roots $v_{b}^{2}>v_{s}^{2}>0$ : $G^{++}$, for $v_{b}^{2}<v_{s}^{2}<$ 0 : $G^{--}$and for $v_{n}^{2}<0<v_{p}^{2}: G^{+-}$denotations will be used, where $v_{b}, v_{s}, v_{n}, v_{p}$ are other names of $v_{1}$ and $v_{2}$ in the cases described.

If we recall definition of $\operatorname{sig}_{Q},(20)$, then without precising the signs of $v^{2}$ we can generally write $G_{0,0}$ as:

$$
G_{0,0}=\frac{2}{\pi}\left(\int_{Q>0} \frac{\operatorname{sign}[f(v)]}{\sqrt{Q(v)}} d v+\int_{Q<0} \frac{1}{\sqrt{Q(v)}} d v\right),
$$

where lower indices at the integrals describe the ranges of integration. It can be proved, that $Q\left(v_{E}\right) \leq 0$, where $v_{E}$ is defined by means of (21), i.e., the point where $f(v)$ changes sign (if it exists), lies outside or on the boundary of the domain of integration of the first integral in (25). That means, that the sign in its numerator is constant within the (connected) domain $Q>0$ and can be transferred in front of the integral. When we define:

$$
I(x, y)=\int_{x}^{y} \frac{1}{\sqrt{Q(v)}} d v
$$

we can express equation (25) as:

$$
G_{0,0}=\frac{2}{\pi}(\operatorname{sign}[f(Q>0)] I(Q>0)+I(Q<0)),
$$

where the conditions in place of the arguments of $I$ functions symbolically denote the ranges of integration. Having $Q(v)=a q(v)$ and with:

$$
\begin{aligned}
q(v) & =\left(v^{2}-v_{1}^{2}\right)\left(v^{2}-v_{2}^{2}\right), \\
J(x, y) & =\int_{x}^{y} \frac{1}{\sqrt{q(v)}} d v,
\end{aligned}
$$

where $v_{1}^{2}$ and $v_{2}^{2}$ are roots (23), we can further simplify integrals $I$ by extracting a constant factor: $I=[J(q>0)+\operatorname{sign}[a] J(q<0)] / \sqrt{a}$ (we choose a branch, where $\sqrt{a}=i \sqrt{|a|}$ for $a<0$; when both $a<0$ and $q<0$ such an extraction changes the sign of the product $1 / \sqrt{a} \sqrt{q})$. If one realizes that $J(q<0)=-i J(-q>0)$ yields imaginary part of $J$, then $I$ can be further simplified to $I=J / \sqrt{a}$ for $a \geq 0$ or $I=J^{*} / \sqrt{a}$ for $a<0$, with the star denoting complex conjugation. Single-equation notation uses step function $\theta$ :

$$
I=\frac{1}{\sqrt{a}}\left(J(q>0)+(-1)^{\theta(-a)} J(q<0)\right) .
$$


Recalling, that the domain $Q>0$ corresponds to the domain of $q>0$ for $a>0$ and of $q<0$ for $a<0$, (30) and (27) allow us to write a compact formula for the Green function, which is deeply connected with the types of solutions of (23):

$$
G_{0,0} \equiv G_{0,0}^{s_{1}, s_{2}}=\frac{2}{\pi \sqrt{a}}\left(s_{n}^{\theta(a)} J\left(q_{n}>0\right)+\left(-s_{m}\right)^{\theta(-a)} J\left(q_{m}<0\right)\right),
$$

where $s_{1}$ and $s_{2}$ are indices denoting the signs of roots (23); on the right-hand side, $s_{n}$ and $s_{m}$ are the signs of the function $f(v)$ in the respective domains $q_{n}>0$ and $q_{m}<0$ of $J$ functions, by which $s_{n}$ and $s_{m}$ are multiplied in (31); indices $n$ and $m$ at $q_{x}$ 's and $s_{x}$ 's are added in case when the given domain $q \gtrless 0$ is multiply connected, to denote Einstein summation convention over parts of the domain (note, that $s_{n}$ and $s_{m}$ should not be confused with $s_{1}$ and $s_{2}$; they are signs of different things).

To proceed we have to examine various possible domains of $q \gtrless 0$. They are bounded by the positive roots (23) of $q(v)$ (note, that the domains consist of the sets of $v$ on the real axis, which may be empty or multiply connected).

When both squared roots (23) $v_{1}^{2}$ and $v_{2}^{2}$ are negative or complex (conjugate) then $q>0$ for all non-negative $v$. Equation (31) yields then:

$$
G_{0,0}^{z}=\beta s_{0}^{\theta(a)} J^{z}(0, \infty),
$$

where $z$ denotes one of the indices: “--" or " $C$ ", $\beta=2 / \pi \sqrt{a}, s_{0}=\operatorname{sign}\left[E+\gamma_{1}-\gamma_{2}+\right.$ $\left.\gamma_{3}\right]=\operatorname{sign}[f(v=0)]$ (also equal to $\left.\operatorname{sign}\left[E+\gamma_{1}\right]\right) . J^{z}(0, \infty)$ is an integral with no singularities in the whole range of integration - this way $G_{0,0}^{z}$ is purely real and positive (for $a>0$ ) or purely imaginary with imaginary part negative $(a<0)$.

When one of the squared roots $(23)$ is positive (denoted as $\left.v_{p}^{2}\right)$, then the integral $J(0, \infty)$ is singular at $v=v_{p}$ and we divide $J$ into two smaller integrals over domains $v \in\left\langle 0, v_{p}\right\rangle$ (corresponding to $q<0$ ) and $v>v_{p}$ (i.e., $q>0$ ). Green function $G^{+-}$is given then as:

$$
G_{0,0}^{+-}=\beta\left(s_{\infty}^{\theta(a)} J\left(v_{p}, \infty\right)+\left(-s_{0}\right)^{\theta(-a)} J^{+-}\left(0, v_{p}\right)\right),
$$

where $s_{0}$ was defined before, $s_{\infty}=\operatorname{sign}[f(v=\infty)]=\operatorname{sign}\left[E+\gamma_{1}+\gamma_{2}-\gamma_{3}\right]$ is (constant) sign of $f(v)$ in the whole range $v \in\left\langle v_{p}, \infty\right)$ (the sign at particular point $v=\infty$ is chosen arbitrarily to simplify the notation).

In case of two positive squared roots: $v_{p 1}^{2}$ and $v_{p 2}^{2}$, integral $J$ has two singularities: at $v_{p 1}$ and $v_{p 2}$ (let's assume $v_{p 1}<v_{p 2}$ ). One of the domains: $q>0$ or $q<0$ consists then of two disconnected parts: $v \in\left\langle 0, v_{p 1}\right\rangle$ and $\left\langle v_{p 2}, \infty\right)$. We have:

$$
\begin{aligned}
G_{0,0}^{++}= & \beta\left(s_{0}^{\theta(a)} J^{++}\left(0, v_{p 1}\right)+s_{\infty}^{\theta(a)} J^{++}\left(v_{p 2}, \infty\right)\right. \\
& \left.+\left(-s_{12}\right)^{\theta(-a)} J^{++}\left(v_{p 1}, v_{p 2}\right)\right),
\end{aligned}
$$

where $s_{12}$ denotes the sign of $f\left(v \in\left\langle v_{p 1}, v_{p 2}\right\rangle\right)$.

Thus we managed to express $G_{0,0}$ by nonsingular integrals $J^{s_{1} s_{2}}(a, b), s_{1}, s_{2} \in\{+,-, C\}$, which can be found in the literature [28]. Eventually the Green function $G_{0,0}$ for any possible combination of squared roots (23) can be expressed as:

$$
\begin{aligned}
G_{0,0}^{C} & =\frac{2}{\pi \sqrt{a}} \frac{1}{\left|v_{c}\right|} s_{0}^{\theta(a)} \mathcal{K}\left(\frac{\Re\left(v_{c}\right)^{2}}{\left|v_{c}\right|^{2}}\right), \\
G_{0,0}^{--} & =\frac{2}{\pi \sqrt{a}} \frac{1}{\left|v_{b}\right|} s_{0}^{\theta(a)} \mathcal{K}\left(1-\frac{v_{s}^{2}}{v_{b}^{2}}\right), \\
G_{0,0}^{+-} & =\frac{2}{\pi \sqrt{a}} \frac{1}{\sqrt{\left|v_{n}^{2}\right|+v_{p}^{2}}}\left[s_{\infty}^{\theta(a)} \mathcal{K}\left(\frac{\left|v_{n}^{2}\right|}{\left|v_{n}^{2}\right|+v_{p}^{2}}\right)\right.
\end{aligned}
$$




$$
\begin{aligned}
& \left.-i\left(-s_{0}\right)^{\theta(-a)} \mathcal{K}\left(\frac{v_{p}^{2}}{\left|v_{n}^{2}\right|+v_{p}^{2}}\right)\right], \\
G_{0,0}^{++}= & \frac{2}{\pi \sqrt{a}} \frac{1}{v_{b}}\left[\left(s_{0}^{\theta(a)}+s_{\infty}^{\theta(a)}\right) \mathcal{K}\left(\frac{v_{s}^{2}}{v_{b}^{2}}\right)\right. \\
& \left.-i\left(-s_{12}\right)^{\theta(-a)} \mathcal{K}\left(1-\frac{v_{s}^{2}}{v_{b}^{2}}\right)\right],
\end{aligned}
$$

where $\mathfrak{R}\left(v_{c}\right)$ is the real part of $v_{c}$ and $\mathcal{F}\left(\alpha, k^{2}\right)$ and $\mathcal{K}\left(k^{2}\right)$ are incomplete and complete elliptic integral of the first kind resp.; a convention of denoting elliptic integrals by the squared moduli is used.

Full $G_{0,0}$ is shown in Figs. 1 and 2. The behavior is representative for LGF's with higher indices as well. The figures show one-particle $\left(\gamma_{4}=0\right)$ and two-particle $\left(\gamma_{4}\right.$ given by (12)) LGF's on $\Gamma-M$ line of square lattice, for $t_{x}<0$ and $t_{2} / t_{x}=-0.3$, values commonly met in HTSC hole-doped materials. For small values of $\mathbf{K}$ the difference between one- and twoparticle LGF's is small but it becomes larger around Brillouin zone corner. With increasing $K$ the two-particle LGF's band becomes wider and, when $K$ becomes large enough, additional peaks appear in $\operatorname{Im}(G)$, at the energies equal to the one-particle's band edges. It may indicate a change in spectral properties of (condensed) pairs described by $G_{0,0}$ around the point $(\pi, \pi)$ in Brillouin zone.

Fig. $1 \operatorname{Re}\left(G_{0,0}\right)$ (thick lines) and $\operatorname{Im}\left(G_{0,0}\right)$ (thin lines) vs $E /\left|t_{x}\right|$ for two-particle function (full lines) and one-particle function (dashed lines) for $t_{x}=t_{y}=-1$ and $t_{2} / t_{x}=-0.3$ at the center of mass momentum $K_{x}=K_{y}=0.75 \pi$

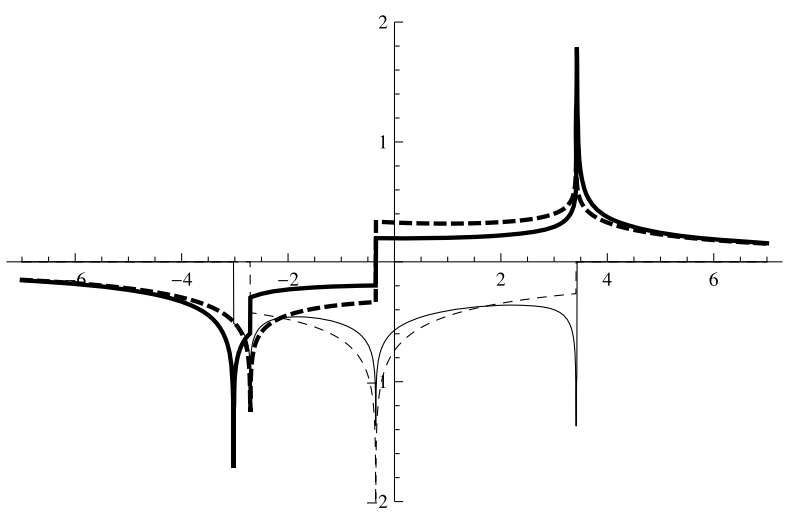

Fig. 2 As in Fig. 1, for the center of mass momentum $K_{x}=K_{y}=0.9 \pi$

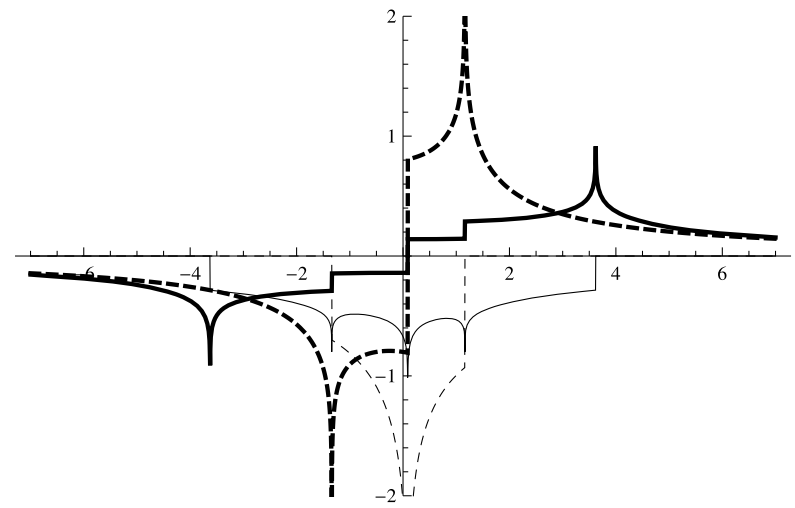




\section{Analytical Formulas for $G_{0,1}$ and $G_{1,1}$}

By the same methods as in the case of $G_{0,0}$ we can calculate nearest-neighbors LGF's: $G_{0,1}$ and $G_{1,0}$ (the latter is given by the same formula as the former but with the coefficients $\gamma_{1} \leftrightarrow \gamma_{2}$ mutually exchanged) and the next-nearest neighbor LGF $G_{1,1}$. After tedious but straightforward calculations the following equations are obtained:

$$
\begin{aligned}
G_{0,1}= & 2 \mathcal{P}(1)-G_{0,0}, \\
G_{1,1}= & -\frac{1}{\gamma_{3}-\gamma_{4}}-\frac{E+\gamma_{2}}{\gamma_{1}-\gamma_{3}} G_{0,0}-\frac{2 \gamma_{2}}{\gamma_{3}-\gamma_{4}} P(1) \\
& +\frac{1}{f_{s}} \frac{1}{2\left(\gamma_{3}-\gamma_{4}\right)}\left(\left(\gamma_{1}-\gamma_{4}\right) \operatorname{sign}\left(\gamma_{1}-\gamma_{3}\right)+\left(\gamma_{1}+\gamma_{4}\right) \operatorname{sign}\left(\gamma_{1}+\gamma_{3}\right)\right) \\
& -\frac{1}{\left(\gamma_{1}^{2}-\gamma_{3}^{2}\right)\left(\gamma_{3}-\gamma_{4}\right)}\left(\left(f_{1} \gamma_{4}-f_{3} \gamma_{1}\right)\left(\mathcal{P}\left(n_{-}\right)+\mathcal{P}\left(n_{+}\right)\right)\right. \\
& \left.+\operatorname{sign}\left(\gamma_{4}\right) \frac{r f_{3}-f_{1} \gamma_{1} \gamma_{4}}{\sqrt{r}}\left(\mathcal{P}\left(n_{-}\right)-\mathcal{P}\left(n_{+}\right)\right)\right),
\end{aligned}
$$

where:

$$
\begin{aligned}
r & =\gamma_{1}^{2}-\gamma_{3}^{2}+\gamma_{4}^{2}, \\
f_{s} & = \begin{cases}\sqrt{r}, & \left|\gamma_{1}\right|>\left|\gamma_{3}\right| \\
\left|\gamma_{4}\right|, & \left|\gamma_{1}\right|<\left|\gamma_{3}\right|,\end{cases} \\
f_{1} & =E \gamma_{1}+\gamma_{2} \gamma_{3}, \\
f_{3} & =E \gamma_{3}+\gamma_{2} \gamma_{1}, \\
n_{+} & =\frac{\left(\gamma_{1}-\gamma_{3}\right)^{2}}{\left(\left|\gamma_{4}\right|+\sqrt{r}\right)^{2}}, \\
n_{-} & =\frac{\left(\gamma_{1}-\gamma_{3}\right)^{2}}{\left(\left|\gamma_{4}\right|-\sqrt{r}\right)^{2}},
\end{aligned}
$$

and $\mathcal{P}(n)$ is an integral analogous to (24):

$$
\mathcal{P}(n)=\frac{2}{\pi} \int_{0}^{\infty} \frac{1}{1+n v^{2}} \frac{\operatorname{sig}_{Q}[f(v)]}{\sqrt{a\left(v^{2}-v_{1}^{2}\right)\left(v^{2}-v_{2}^{2}\right)}},
$$

where $v_{1}^{2}$ and $v_{2}^{2}$ are given by (23). Note, that $n_{ \pm}$are either complex or nonnegative, so integral $\mathcal{P}(n)$ has the same singularities as $G_{0,0}$. Also the way of calculating is the same: we introduce integrals $I(n, x, y)=\int_{x}^{y} d v /\left(1+n v^{2}\right) \sqrt{Q(v)}$ and $J(n, x, y)=\int_{x}^{y} d v /(1+$ $\left.n v^{2}\right) \sqrt{q(v)}$ analogous to (26) and (29). With the exchange of $I$ into $I(n)$ and $J$ into $J(n)$ equation (30) remains true and $\mathcal{P}(n)$ is given by the right-hand side of (27). Thus the solutions have the same structure as in the case of $G_{0,0}$ and the previously derived formulas can be used. When we introduce integrals $\mathcal{P}^{z}(n)$, where $z$ is one of the indices from the set: [ " $C$ ", “++", “+-", “--"\} we can express $\mathcal{P}^{z}(n)$ 's by the right-hand sides of (32), (33), (34) for $G_{0,0}^{z}$, where new functions $J^{z}(n)$ 's are used instead of $J^{z}$ 's. All functions $J^{z}(n)$ can be expressed by elliptic integrals and can be found in literature [28], except $J^{C}(n)$ for complex roots (23):

$$
\begin{aligned}
J^{C}(n, 0, \infty)= & \frac{2}{\Im\left(v_{c}\right)} \frac{1}{1+B} \\
& \times\left[\hat{J}(0,1)+\frac{B(1-B)}{\sqrt{-B(B-1)^{2}}}\left(\hat{J}\left(\frac{-1}{y_{+}}, 0,1\right)-\hat{J}\left(\frac{-1}{y_{-}}, 0,1\right)\right)\right],
\end{aligned}
$$


where:

$$
\begin{aligned}
& \hat{J}(x, y)=\hat{J}(0, x, y), \\
& \hat{J}(n, x, y)=J^{v_{a}^{2}, 1 / v_{a}^{2}}(n, x, y), \\
& J^{a, b}(n, x, y)=\int_{x}^{y} \frac{1}{1+n v^{2}} \frac{d v}{\sqrt{\left(v^{2}-a\right)\left(v^{2}-b\right)}}, \\
& v_{a}^{2}=-\frac{\left|v_{c}\right|+\left|\Re\left(v_{c}\right)\right|}{\left|v_{c}\right|-\left|\Re\left(v_{c}\right)\right|}, \\
& y_{ \pm}=1-\frac{8 B}{(B+1)^{2}} \pm \frac{4 \sqrt{-B(B-1)^{2}}}{(B+1)^{2}}, \\
& B=n\left|v_{c}^{2}\right| .
\end{aligned}
$$

Because integrals (49) and (50) can also be found in [28] thus we managed to express $G_{0,1}$ and $G_{1,1}$ by elliptic integrals of the first, second and third kinds. The list of needed $J^{z}(n)$ 's is given in the Appendix.

\section{Recursion Formulae}

$G_{0,0}$ can be used to obtain the density of states or the energy of bound pairs in the Hubbard model. Yet calculating the wave function on various lattice sites or properties of pairs in extended Hubbard models requires often $G_{m, n}$ for other values of $m$ 's and $n$ 's. As the analytic formulae for $G_{m, n}$ become more and more complicated with growing $|m|$ and $|n|$ the recursion formulae are of interest. The basic one is equation defining the Green function:

$$
\begin{aligned}
2 E G_{m, n}= & 2 \delta_{m, 0} \delta_{n, 0}+\gamma_{1}\left(G_{m+1, n}+G_{m-1, n}\right)+\gamma_{2}\left(G_{m, n+1}+G_{m, n-1}\right) \\
& +\frac{\gamma_{3}}{2}\left(G_{m+1, n-1}+G_{m-1, n+1}+G_{m+1, n+1}+G_{m-1, n-1}\right) \\
& +\frac{\gamma_{4}}{2}\left(G_{m+1, n-1}+G_{m-1, n+1}-G_{m+1, n+1}-G_{m-1, n-1}\right) .
\end{aligned}
$$

As this equation is true for every $E$ one may differentiate it by $E$ and the equality will still hold. After such a procedure we get:

$$
\begin{aligned}
2 G_{m, n}= & -2 E G_{m, n}^{\prime}+\gamma_{1}\left(G_{m+1, n}^{\prime}+G_{m-1, n}^{\prime}\right)+\gamma_{2}\left(G_{m, n+1}^{\prime}+G_{m, n-1}^{\prime}\right) \\
& +\frac{\gamma_{3}}{2}\left(G_{m+1, n+1}^{\prime}+G_{m-1, n-1}^{\prime}+G_{m+1, n-1}^{\prime}+G_{m-1, n+1}^{\prime}\right) \\
& +\frac{\gamma_{4}}{2}\left(G_{m+1, n-1}^{\prime}+G_{m-1, n+1}^{\prime}-G_{m+1, n+1}^{\prime}-G_{m-1, n-1}^{\prime}\right),
\end{aligned}
$$

where $G_{m, n}^{\prime}$ means $d G_{m, n}(E) / d E$ and is given by equation similar to (13), just with the denominator squared and minus in front.

By integration by parts of one of the integrals in (13) we can get another type of relations involving derivatives [29]:

$$
\begin{aligned}
2 m G_{m, n}= & \gamma_{1}\left(G_{m+1, n}^{\prime}-G_{m-1, n}^{\prime}\right) \\
& +\frac{\gamma_{3}}{2}\left(G_{m+1, n+1}^{\prime}+G_{m+1, n-1}^{\prime}-G_{m-1, n+1}^{\prime}-G_{m-1, n-1}^{\prime}\right) \\
& +\frac{\gamma_{4}}{2}\left(G_{m-1, n-1}^{\prime}+G_{m+1, n-1}^{\prime}-G_{m-1, n+1}^{\prime}-G_{m+1, n+1}^{\prime}\right)
\end{aligned}
$$




$$
\begin{aligned}
2 n G_{m, n}= & \gamma_{2}\left(G_{m, n+1}^{\prime}-G_{m, n-1}^{\prime}\right) \\
& +\frac{\gamma_{3}}{2}\left(G_{m+1, n+1}^{\prime}+G_{m-1, n+1}^{\prime}-G_{m+1, n-1}^{\prime}-G_{m-1, n-1}^{\prime}\right) \\
& +\frac{\gamma_{4}}{2}\left(G_{m-1, n+1}^{\prime}+G_{m-1, n-1}^{\prime}-G_{m+1, n-1}^{\prime}-G_{m+1, n+1}^{\prime}\right) .
\end{aligned}
$$

Procedure described in [26] enables us to obtain yet another set of recursion formulae valid on coordinate axes in direct space (i.e., for one of indices equal to zero):

$$
\begin{aligned}
& 2 n\left(\gamma_{1}^{2}+\frac{1}{2}\left(-\gamma_{2}^{2}+\gamma_{3}^{2}+\gamma_{4}^{2}\right)-E^{2}\right) G_{0, n} \\
& \quad+\left(\gamma_{1} \gamma_{3}+\gamma_{2} E\right)\left((2 n+1) G_{0, n+1}+(2 n-1) G_{0, n-1}\right) \\
& \quad+\frac{1}{2}\left(-\gamma_{2}^{2}+\gamma_{3}^{2}-\gamma_{4}^{2}\right)\left((n+1) G_{0, n+2}+(n-1) G_{0, n-2}\right)=0, \\
& 2 m\left(\gamma_{2}^{2}+\frac{1}{2}\left(-\gamma_{1}^{2}+\gamma_{3}^{2}+\gamma_{4}^{2}\right)-E^{2}\right) G_{m, 0} \\
& \quad+\left(\gamma_{2} \gamma_{3}+\gamma_{1} E\right)\left((2 m+1) G_{m+1,0}+(2 m-1) G_{m-1,0}\right) \\
& \quad+\frac{1}{2}\left(-\gamma_{1}^{2}+\gamma_{3}^{2}-\gamma_{4}^{2}\right)\left((m+1) G_{m+2,0}+(m-1) G_{m-2,0}\right)=0,
\end{aligned}
$$

and the derivatives:

$$
\begin{aligned}
4 E n G_{0, n}-\gamma_{2}\left((2 n+1) G_{0, n+1}+(2 n-1) G_{0, n-1}\right) \\
=2 n\left(\gamma_{1}^{2}+\frac{1}{2}\left(-\gamma_{2}+\gamma_{3}^{2}+\gamma_{4}^{2}\right)-E^{2}\right) G_{0, n}^{\prime} \\
\quad+\left(\gamma_{1} \gamma_{3}+\gamma_{2} E\right)\left((2 n+1) G_{0, n+1}^{\prime}+(2 n-1) G_{0, n-1}^{\prime}\right) \\
\quad+\frac{1}{2}\left(-\gamma_{2}^{2}+\gamma_{3}^{2}-\gamma_{4}^{2}\right)\left((n+1) G_{0, n+2}^{\prime}+(n-1) G_{0, n-2}^{\prime}\right) \\
4 E m G_{m, 0}-\gamma_{1}\left((2 m+1) G_{m+1,0}+(2 m-1) G_{m-1,0}\right) \\
=2 m\left(\gamma_{2}^{2}+\frac{1}{2}\left(-\gamma_{1}^{2}+\gamma_{3}^{2}+\gamma_{4}^{2}\right)-E^{2}\right) G_{m, 0}^{\prime} \\
\quad+\left(\gamma_{2} \gamma_{3}+\gamma_{1} E\right)\left((2 m+1) G_{m+1,0}^{\prime}+(2 m-1) G_{m-1,0}^{\prime}\right) \\
\quad+\frac{1}{2}\left(-\gamma_{1}^{2}+\gamma_{3}^{2}-\gamma_{4}^{2}\right)\left((m+1) G_{m+2,0}^{\prime}+(m-1) G_{m-2,0}^{\prime}\right) .
\end{aligned}
$$

\section{Solution for any $G_{m, n}$}

Recursive relations enable us to express "new" functions, with the "higher" indices by the "old" ones, with "lower" indices. They are useful if we know all the "old" functions or, equivalently, if the number of "new" functions is not too large. As equation (55) shows, LGF for given $m$ and $n$ connects to eight other LGF's, so, unlike the case with nearestneighbor hopping only, it is impossible to form a closed set of equations of "pure" LGF's of the type (55), like it was done in other works on LGF's [26].

We may try to use symmetry conditions:

$$
G_{-m,-n}=G_{m, n},
$$


Fig. 3 Indices of sites belonging to the first three layers on rectangular lattice: "zeroth" layer-just the site $\{0,0\}$, first layer-indices grayed out, second layer-indices boxed, indices blacked out are excluded due to symmetry reasons

\begin{tabular}{|l|l|l|}
\hline$\{0,2\}$ & $\{1,2\}$ & $\{2,2\}$ \\
\hline$\{0,1\}$ & $\{1,1\}$ & $\{2,1\}$ \\
$\{0,0\}$ & $\{1,0\}$ & $\{2,0\}$ \\
\cline { 3 - 4 } & $\{1,-1\}$ & $\{2,-1\}$ \\
\hline & $\{1,-2\}$ & $\{2,-2\}$ \\
\hline
\end{tabular}

which decrease the amount of "new" functions by half (let's say to the ones with the first or both indices positive), but even that is not enough to close the system of equations of the type (55).

Let's note, that formulas (56)-(58) introduce many derivatives of LGF's but no additional "pure" LGF's. This way we get additional, indirect relations among "pure" LGF's and we may hope, that the joint system of equations for $G_{m, n}$ 's and $G_{m, n}^{\prime}$ 's will close.

Layers Indeed, it turns out, that the LGF's arranged in carefully selected sets, which we call layers, do close. The LGF's indices correspond to the sites of the lattice and the $z$ 'th layer consist of lattice sites, which are " $\langle\langle z\rangle\rangle$ sites apart from the site $(0,0)$ " and belong to different Green functions. The sites with both indices non-positive are omitted (except the site $(0,0))$ due to symmetry relation $(63)$. The first three layers are shown in Figure 3 . The "zeroth" layer consists of just one site $(0,0)$. The first layer is created of the sites adjacent to the site $(0,0)$, the second layer-sites adjacent to the first (from the "outside") etc.

Example equation of the type (55) for $G_{0,0}$ ("zeroth" layer) together with derivative equations of the types (56)-(58) for $G_{m, n}$ 's from the zeroth and the first layers form a system of 16 equations with 18 variables (Green functions and their derivatives). Only 13 of these 16 equations are linearly independent, so we finally need five parameters to solve the system, e.g.: $G_{0,0}, G_{0,0}^{\prime}, G_{0,1}, G_{1,0}$ and $G_{1,1}$ (other choices of parameters are also possible, in fact many others). This solution yields enough known parameters to solve the analogous system of equations for the next layers. In general, (56)-(58) for $G_{m, n}$ 's from within the $z$ 'th layer mix those $G_{m, n}$ 's with $G_{m, n}^{\prime}$ 's from layers $z-1, z, z+1$, so we can use the results of previous layers to solve the next. Starting from the second layer the such-obtained systems of equations are closed and even overcomplete and no additional parameters are needed to find their solution. Thus the knowledge of five above-mentioned parameters is sufficient, in principle, to find Green functions on the entire lattice.

\section{Discussion and Conclusions}

Let's note, that every LGF admits also a nonphysical, diverging solution. Because a linear combination of solutions is also an LGF solution, numerical value of even the physically correct Green function contains a small admixture of nonphysical LGF, due to unavoidable numerical errors. When calculated recursively this small error grows to dominate the solutions for large enough LGF's indices [30]. 
Recursion relations (59)-(62), for LGF's with one of the indices zero, are presented for the sake of completeness only, they are not used to make a closed system of equations. The author did not find the way to assemble a system, which would enable to calculate $G_{m, n>0}$ based on the knowledge of $G_{m, 0}$ as done in Ref. [26] in case of nn hopping in 3d.

The one-particle and two-particle LGF's are the same (up to a constant " 2 ") only for the center of mass momentum zero. The differences between them become large near the corner of Brillouin zone.

The correctness of analytical formulae was checked numerically by direct integration of (13) for $E$ outside the band and, in case of the imaginary part of $G_{m, n}$-for $E$ within the band-by direct integration of (24) and (47) and by direct summing of Dirac delta functions appearing after adding an infinitesimal imaginary part to $E$. Note, that for $E \rightarrow \pm \infty$, coefficient $a$, (16), becomes positive and both roots (23) become negative or complex. Thus the sign of $G_{0,0}(E \rightarrow \pm \infty)$ is equal to $s_{0} \sim \operatorname{sign}[E]$ in agreement with (2). Also, for any roots (23), the imaginary part of $G_{0,0}$ turns out to be always of the same sign-negative-this is correct, as $\Im\left(G_{0,0}\right)$ is proportional to the density of two-particle states.

In conclusion present paper shows exact analytical formulas for the two-particle lattice Green functions: on the same site $G_{0,0}$, for the nearest neighbors $G_{0,1}, G_{1,0}$ and next-nearest neighbor $G_{1,1}$ of the rectangular, two-dimensional lattice, including full wave vector dependence on the center-of-mass momentum and for arbitrary energy. With the formula for $G_{0,0}$ the derivative $G_{0,0}^{\prime}$ can also be easily calculated and with that amount of known functions one can obtain LGF's on any lattice site via the recursion relations, assembled in a novel way with an extensive use of LGF's derivatives. Five kind of new recursion relations are presented, including a defining difference equation for $G_{m, n}$ and recursion relations containing derivatives. Large differences between one- and two-particle LGF's are found for center-of-mass momenta $\mathbf{K}$ close to $(\pi, \pi)$.

Open Access This article is distributed under the terms of the Creative Commons Attribution Noncommercial License which permits any noncommercial use, distribution, and reproduction in any medium, provided the original author(s) and source are credited.

\section{Appendix}

Explicit formulae for integrals $J^{z}(n)$ are presented for completeness.

$$
\begin{aligned}
J^{--}(n, 0, \infty) & =\frac{1}{\left|v_{b}\right|} \frac{1}{n\left|v_{s}^{2}\right|-1}\left[n\left|v_{s}^{2}\right| \Pi\left(1-n\left|v_{s}^{2}\right|, 1-\frac{v_{s}^{2}}{v_{b}^{2}}\right)-\mathcal{K}\left(1-\frac{v_{s}^{2}}{v_{b}^{2}}\right)\right], \\
J^{+-}\left(n, 0, v_{p}\right) & =\frac{-i}{\sqrt{\left|v_{n}^{2}\right|+v_{p}^{2}}} \frac{1}{1+n v_{p}^{2}} \Pi\left(\frac{n v_{p}^{2}}{1+n v_{p}^{2}}, \frac{v_{p}^{2}}{\left|v_{n}^{2}\right|+v_{p}^{2}}\right), \\
J^{+-}\left(n, v_{p}, \infty\right) & =\frac{1}{\sqrt{\left|v_{n}^{2}\right|+v_{p}^{2}}}\left[\mathcal{K}\left(\frac{\left|v_{n}^{2}\right|}{\left|v_{n}^{2}\right|+v_{p}^{2}}\right)-\frac{n v_{p}^{2}}{1+n v_{p}^{2}} \Pi\left(\frac{1}{1+n v_{p}^{2}}, \frac{\left|v_{n}^{2}\right|}{\left|v_{n}^{2}\right|+v_{p}^{2}}\right)\right], \\
J^{++}\left(n, 0, v_{s}\right) & =\frac{1}{v_{b}} \Pi\left(-n v_{s}^{2}, \frac{v_{s}^{2}}{v_{b}^{2}}\right), \\
J^{++}\left(n, v_{s}, v_{b}\right) & =\frac{-i}{v_{b}} \frac{1}{1+n v_{b}^{2}} \Pi\left(\frac{n v_{b}^{2}}{1+n v_{b}^{2}}\left(1-\frac{v_{s}^{2}}{v_{b}^{2}}\right), 1-\frac{v_{s}^{2}}{v_{b}^{2}}\right), \\
J^{++}\left(n, v_{b}, \infty\right) & =\frac{1}{v_{b}}\left[\mathcal{K}\left(\frac{v_{s}^{2}}{v_{b}^{2}}\right)-\Pi\left(\frac{-1}{n v_{b}^{2}}, \frac{v_{s}^{2}}{v_{b}^{2}}\right)\right],
\end{aligned}
$$


where $\Pi\left(n, \psi, k^{2}\right)$ is elliptic integral of the third kind:

$$
\Pi\left(n, \psi, k^{2}\right)=\int_{0}^{\psi} \frac{1}{1-n \sin ^{2} \phi} \frac{1}{\sqrt{1-k^{2} \sin ^{2} \phi}} d \phi,
$$

and $\Pi\left(n, k^{2}\right)=\Pi\left(n, \pi / 2, k^{2}\right)$.

$J^{C}(n),(48)$, needs two integrals, defined in the text, calculated for $v_{b}^{2}>v_{s}^{2}>0$ :

$$
\begin{aligned}
J^{-v_{s}^{2},-v_{b}^{2}}(0, u)= & \frac{1}{v_{b}} \mathcal{F}\left(\tan ^{-1} \frac{u}{v_{s}}, 1-\frac{v_{s}^{2}}{v_{b}^{2}}\right), \\
J^{-v_{s}^{2},-v_{b}^{2}}(n, 0, u)= & \frac{1}{v_{b}} \frac{1}{n v_{s}^{2}-1}\left[n v_{s}^{2} \Pi\left(1-n v_{s}^{2}, \tan ^{-1} \frac{u}{v_{s}}, 1-\frac{v_{s}^{2}}{v_{b}^{2}}\right)\right. \\
& \left.-\mathcal{F}\left(\tan ^{-1} \frac{u}{v_{s}}, 1-\frac{v_{s}^{2}}{v_{b}^{2}}\right)\right] .
\end{aligned}
$$

\section{References}

1. Economou, E.: Green's Functions in Quantum Physics. Springer, Berlin (2006)

2. Watson, G.N.: Three triple integrals. Q. J. Math. Oxford 10, 266-276 (1939)

3. Wortis, M.: Bound states of two spin waves in the Heisenberg ferromagnet. Phys. Rev. 132, 85 (1963)

4. Hughes, D.: Random Walks and Random Environments, vol. 1. Clarendon Press, Oxford (1995)

5. Montet, G.: Integral methods in the calculation of correlation factors in diffusion. Phys. Rev. B 3, 650 (1973)

6. Wolfram, T., Callaway, J.: Spin-wave impurity states in ferromagnets. Phys. Rev. 130, 2207 (1963)

7. Montroll, E.W.: Dynamics of a square lattice. J. Chem. Phys. 15, 575 (1947)

8. Dalton, N.W., Wood, D.W.: Critical behaviour of the simple anisotropic Heisenberg model. Proc. Phys. Soc. 90, 459 (1967)

9. Koster, G.F., Slater, D.C.: Simplified impurity calculation. Phys. Rev. 96, 1208 (1954)

10. Cserti, J.: Application of the lattice Green's function for calculating the resistance of an infinite network of resistors. Am. J. Phys. 68, 896 (2000)

11. Asad, J.H., Hijjawi, R.S., Sakaji, A., Khalifeh, J.M.: Remarks on perturbation of infinite networks of identical resistors. Int. J. Theor. Phys. 44(4), 3977 (2005)

12. Asad, J.H., Hijjawi, R.S., Sakaji, A., Khalifeh, J.M.: Infinite network of identical capacitors by Green's function. Int. J. Mod. Phys. B 19(24), 3713-3721 (2005)

13. Hijjawi, R.S., Asad, J.H., Sakaji, A.J., Khalifeh, J.M.: Perturbation of an infinite network of identical capacitors. Int. J. Mod. Phys. B 21(2), 199-209 (2007)

14. Micnas, R., Ranninger, J., Robaszkiewicz, S.: Superconductivity in narrow-band systems with local nonretarded attractive interactions. Rev. Mod. Phys. 62, 113 (1990)

15. Bak, M., Micnas, R.: Extended bound states of fermions on $2 \mathrm{~d}$ square lattice beyond the nn hopping and interactions. Mol. Phys. Rep. 24, 168 (1999). arXiv:cond-mat/9909089

16. Blaer, A.S., Ren, H.C., Tchernyshyov, O.: Extended bound states and resonances of two fermions on a periodic lattice. Phys. Rev. B 55, 6035 (1997)

17. Lin, H.Q.: Dilute gas of electron pairs in the $t-J$ model. Phys. Rev. B 44, 4674 (1991)

18. Kornilovitch, P.: Enhanced stability of bound pairs at nonzero lattice momenta. Phys. Rev. B 69, 235110 (2004)

19. Randeria, M., Duan, J.-M., Shieh, L.-I.: Bound states, cooper pairing, and Bose condensation in two dimensions. Phys. Rev. Lett. 62, 981 (1989)

20. Kadolkar, C.Y., Basu, S.: Phase diagram of the t-J model on a honeycomb lattice. Phys. Rev. B 73, 104501 (2006)

21. Katsura, S., Morita, T., Inawashiro, S., Horiguchi, T., Abe, Y.: Lattice Green's functions. Introduction. J. Math. Phys. 12, 892 (1971)

22. Hijjawi, R.S., Asad, J.H., Sakaji, A., Khalifeh, J.M.: Lattice Green's function for the face centered cubic lattice. Int. J. Theor. Phys. 43, 2299 (2004)

23. Xing, D. Y., Liu, M., Gong, C.D.: Van Hove singularity and isotope effect in high- $T_{c}$ copper oxides. Phys. Rev. B 44, 12525 (1991) 
24. Polak, T.P., Kopec, T.K.: Superfluid to Mott-insulator transition in an anisotropic two-dimensional optical lattice. Ann. Phys. 17, 947 (2008)

25. Bak, M.: Lattice Green functions on a two-dimensional rectangular lattice with next-nearest neighbor interaction. Acta Phys. Pol. A 118, 386 (2010)

26. Morita, T.: Use of recurrence formula in computing the lattice Green function. J. Phys. A, Math. Gen. 8, 478 (1975)

27. Katsura, S., Inawashiro, S.: Lattice Green's functions for the rectangular and the square lattices at arbitrary points. J. Math. Phys. 12, 1622 (1971)

28. Byrd, P.E., Friedman, M.D.: Handbook of Elliptic Integrals for Engineers and Physicists. Springer, Berlin (1954)

29. Horiguchi, T., Morita, T.: Note on the lattice Green's function for the simple cubic lattice. J. Phys. C, Solid State Phys. 8, L232 (1975)

30. Berciu, M.: On computing the square lattice Green's function without any integrations. J. Phys. A, Math. Theor. 42, 395207 (2009) 